\title{
Is PECAM-1 a Mechanoresponsive Molecule?
}

\author{
Keigi Fujiwara $^{1 *}$, Michitaka Masuda², Masaki Osawa², Yumiko Kano², and Kazuo Katoh² \\ ${ }^{1}$ Center for Cardiovascular Research, Box 679, Department of Medicine, University of Rochester, Rochester, \\ NY 14624, USA, ${ }^{2}$ Department of Structural Analysis, National Cardiovascular Center Research Institute, Suita, \\ Osaka 565-8565 Japan
}

\begin{abstract}
Endothelial cells are capable of responding to fluid shear stress, but the molecular mechanism for this biological response remains largely unknown. Our studies indicate that the cell-cell adhesion site is a possible site of flow sensing. PECAM-1, a cell adhesion molecule localized to the interendothelial cell adhesion site, is tyrosine-phosphorylated when endothelial cells are exposed to physiological levels of fluid shear stress. This PECAM-1 phosphorylation initiates a signaling cascade leading to ERK activation. Here we review what is known about PECAM-1 tyrosine phosphorylation and suggest a possible role of PECAM-1 in mechanosensing by endothelial cells.
\end{abstract}

Key words: PECAM-1/fluid shear stress/mechanosensing/cell adhesion/cytoskeleton

Until recently, blood vessels were considered to be a static hardware of the body that distributed blood throughout the body, and their biology was not a major concern for biologists and medical researchers. When blood vessels were referred to as 'living pipes', it meant that they were made of live cells and could be repaired if damaged. However, recent progress in vascular biology gave a new meaning to the term. We now know that new vessels can be formed while existing ones can be remodeled. We also know that they are metabolically highly active and perform multifaceted functions, such as synthesizing physiologically active substances, receiving and transmitting chemical as well as mechanical signals, and controlling the passage of molecules and cells across the vessel wall. The blood vessel has become a target of intense research also because its diseases are often life-threatening.

Atherosclerosis is a disease that affects the arteries. It has

\footnotetext{
* To whom correspondence should be addressed: Cener for Cardiovascular Research, Box 679, Department of Medicine, University of Rochester, Rochester, NY 14624, USA.

Tel: +1-716-273-5714, Fax: +1-716-273-1497

E-mail: Keigi_Fujiwara@urmc.rochester.edu

Abbreviations: PECAM-1, endothelial cell adhesion molecule-1; eNOS, endothelial nitric oxide synthase; ERK, extracellular-signal regulated kinase; FAK, focal adhesion kisase; MAP, mitogen activated protein; NO, nitric oxide; TGF- $\beta$, transforming growth factor- $\beta$; SHP-2, SH2-protein tyrosine phosphatase; SH2, Src-homology 2; PKC, protein kinase C; PMA, phorbol myristate acetate; MEK, MAP kinase-ERK kinase; VEGF, vascular endothelial growth factor; Ig, Immunoglobulin; IgG, Immunoglobulin
} G. long been known that certain regions within the arterial tree are more likely to develop atherosclerotic lesions. The most common region is the branch point of the artery. Many factors are known to contribute to the development and progression of the disease, such as consumption of fatty foods, smoking, stress, age, male hormone, high blood sugar, and lack of exercise. However, it is not obvious how these factors that most likely affect the entire body contribute to the development of atherosclerosis in specific areas of the blood vessel. Attempting to find some features specific to the vessel branch points, investigators turned to hemodynamic forces of blood flow. The blood flow pattern within the straight portion of arteries is considered to be essentially laminar, but disturbed flow is expected in the areas of high incidence of atherogenesis (Caro et al., 1971; Zarins et al., 1983). In terms of fluid shear stress, the vessel wall of the area with disturbed flow is exposed to more reduced shear stress than the straight portion of the vessel. Thus, it is widely accepted that the reduced level of time-averaged fluid shear stress in large arteries is pro-atherogenic (for review, see Gimbrone, 1999).

Conversely, the areas exposed to steady laminar flow of high shear stress have a lower tendency to develop plaques, and the current thinking is that steady laminar flow is atheroprotective (for review, see Traub and Berk, 1997). This notion is supported by the presence of many flow-induced responses that can be atheroprotective. For example, fluid shear stress-induced production of NO, which is 
known to be involved in regulating platelet aggregation, leukocyte adhesion, lipoprotein metabolism and vascular tone, is thought to play an important role in this regard (Traub and Berk, 1997). Flow-induced NO production appears to be achieved first by increased $\mathrm{Ca}^{2+}$ in the cell, which rapidly activates eNOS activity, followed by eNOS phosphorylation by Akt (Dimmeler et al., 1999), which provide a $\mathrm{Ca}^{2+}$-independent and more sustained eNOS activity, and then by increased transcription of eNOS gene (Topper et al., 1996). In addition, endothelial cells exposed to laminar shear stress show decreased expression of adhesion molecules, which is anti-inflammatory (Ohtsuka et al., 1993), increased production of prostacyclin, which is antithrombotic (Okahara et al., 1998), and increased expression of TGF- $\beta$, which is inhibitory for smooth muscle cell growth (Ueba et al., 1997). By eliciting all of these responses simultaneously in endothelial cells, fluid shear stress is expected to provide powerful protection against atherogenesis.

Fluid shear stress is the frictional force acting on the luminal surface of endothelial cells. Although in vitro as well as in vivo studies have established that it affects endothelial cells' structure, biosynthetic activity, physiology and gene expression (for review, see Davies, 1995), it is not known how these cells sense fluid shear stress. In this brief review, we will discuss a possible flow sensing mechanism present in endothelial cells.

\section{Early flow responses of endothelial cells}

The classical studies by Fry (1968), Flaherty et al. (1972), and Silkworth et al. (1975) have clearly demonstrated that blood flow is a determining factor for the axial (i.e. parallel to the direction of blood flow) organization of endothelial cell morphology, indicating that these cells have a mechanism for sensing fluid flow. It is now established that fluid flow influences not only the structure but also the biosynthetic activities, gene expressions, and physiology of endothelial cells (Davies, 1995). Recent studies indicate that both temporal (Bao et al., 1999) and spatial (Nagel et al., 1999) gradients of shear stress are important parameters of flow that cause the expression and accumulation in the nucleus of transcription factors such as egr-1 and c-fos. Our current knowledge on the molecular mechanism(s) of flow sensing is severely limited. Most analyses have been done using in vitro systems in which confluent endothelial cell cultures are exposed to flow of culture medium. In such experiments, one expects the flow sensing mechanisms to be activated soon after flow is applied to the cell.

The early flow-dependent events include activation of ion channels. There are many types of ion channels in endothelial cells, and some of them are activated by fluid shear stress (Nilius et al., 1997). Olsen et al. (1988) reported that shear stress activated a $\mathrm{K}^{+}$channel in cultured endothelial cells and that it could be a mechanosensor of these cells. Schwartz et al. (1992) reported that a cation channel, which was more permeable to $\mathrm{Ca}^{2+}$ than to $\mathrm{Na}^{+}$, was activated by shear stress. Increased intracellular $\mathrm{Ca}^{2+}$ is a well-established early flow response of endothelial cells (Ando et al., 1988). This could be achieved by increased activity of the cation channel, but flow-stimulated PI turnover appears to play a major role (Mo et al., 1991; Dull and Davies, 1991; Shen et al., 1992). Whether or not the $\mathrm{K}^{+}$ channel activation and $\mathrm{Ca}^{2+}$ mobilization are causally related is not fully known, but they appear to be independent events (Schilling et al., 1992). In certain prokaryotic and eukaryotic cells, activation of stretch sensitive ion channels is tied to mechanosensing (Sukharev et al., 1994; Kanzaki et al., 1999). However, it is not known if endothelial cells have homologues of these channels. Although the presence of stretch-activated ion channels is reported (for review, see Nilius, 1997), their molecular identity has not been worked out. Several important questions remain to be answered. One is the mechanism by which fluid shear stress activates these ion channels. Another is the precise localization of these channels on the cell surface. This information may provide a clue to solving the first question. The third and most important point is to know if and how these ion channel activities are related to the flow-induced morphological, biochemical, and physiological changes in endothelial cells.

\section{Possible sites of mechanosensing}

Mechanical stresses act on the part of the cell that resists deformation by external forces. Fluid shear stress is the frictional force that acts on the apical surface of endothelial cell. Thus, one might hypothesize a flow sensing apparatus to be in or near the part of the cell membrane that resists deformation by fluid shear stress. The areas of the plasma membrane where the cytoskeleton is anchored may be such domains, and there are several candidate areas in endothelial cells. The apical plaque is a site where the apical stress fibers are anchored to the apical plasma membrane and are present in both cultured (Katoh et al., 1995) and in situ (Kano et al., 1996) endothelial cells. Although only limited information on the molecular composition of the structure is available, our studies so far have revealed little evidence (such as localization of signaling molecules) that the apical plaque is a site of mechanosignal transduction. Because of its location on the cell surface, which is ideal for flow sensing, this unique structure needs to be further investigated in the light of mechanosignal transduction.

Focal adhesions and cell-cell adhesion sites are other membrane associated regions that may resist deformation, thus are possible sites for mechanosignal transduction. Indeed, certain molecules involved in signal transduction have been localized to these sites (Dejana, 1997; Shattil and Ginsberg, 1997; Buckley et al., 1998; Dejana et al., 1999; Kano et al., 2000). Anatomically, however, these regions are not directly exposed to fluid flow. Thus, if these sites are 
involved in shear stress sensing and subsequent signal transduction, there must be some (cytoskeletal) structures that link these regions to the apical cell surface. Such a structure needs to be firmly anchored to the apical surface of the cell. It must also be reasonably rigid so that it can transmit the force of shear stress with little loss to these cell adhesion sites. Recently, the vimentin intermediate filament system was proposed to be an important cytoskeletal structure involved in mechanotransduction in endothelial cells (Helmke et al., 2000). Whether or not intermediate filaments are firmly anchored to the apical cell membrane is not known. It is also unknown if the intermediate filament system is firm enough to effectively transmit force to specific regions of a cell.

Our recent studies indicate that the apical plaque is not an isolated, esoteric structure of a cell but is an integral part of the three dimensional network of the stress fiber system. The apical stress fibers are firmly anchored to the apical plasma membrane at the apical plaque and run between the apical plaque and either the focal adhesion (Katoh et al., 1995) or the lateral cell-cell adhesion site (Kano et al., 2000). Stress fibers are sarcomere-like structures, and we have recently demonstrated their contractile property (Katoh et al., 1998, 2000). By isometrically contracting, stress fibers in the cell are likely to be under tension, as several workers have suggested (for review, see Byers et al., 1984), and to be relatively stiff. As such, stress fibers are ideal structures for transmitting mechanical forces from one point of a cell to another. Thus, it is feasible that the force of fluid shear stress is transmitted from the apical plaque to the focal adhesion and the lateral cell-cell association site via the apical stress fibers. Both of these adhesion sites are areas of cells that are fixed and, therefore, mechanical forces can act on them. We suggest that these cell adhesion points are possible sites where fluid shear stress sensing and subsequent signal transduction could take place (Kano et al., 2000), and indeed other investigators have also advanced a similar hypothesis (Davies, 1995).

There is evidence suggesting that some flow-dependent signaling events are occurring at these adhesion sites even though they are not directly exposed to flow. For example, integrins localized at the focal adhesion were able to transduce mechanical forces (Wang et al., 1993). FAK and c-Src are kinases associated with the focal adhesion and are phosphorylated when cultured endothelial cells are exposed to fluid shear stress (Tseng et al., 1995; Ishida et al., 1996). These authors suggested the presence of a MAP kinase activation pathway that depends on cell adhesion and fluid shear stress. Flow induced vasodilation was inhibited when coronary arterioles were treated with RGD peptides or blocking antibodies against $\beta 3$-integrin (Muller et al., 1997). The hypothesis also predicts flow-dependent activation of signaling molecules at the lateral cell adhesion. Immunolocalization studies have shown that a number of signaling molecules, including Src, Lyn, Yes, Csk, $\beta$-cate- nin, plakoglobin, components of the Ras signaling pathway, small GTP binding proteins, and phospho-tyrosine containing proteins are present at the interendothelial cell adhesion (Dejana, 1997; Kano et al., 2000). The presence of these molecules suggests signaling activity at the endothelial cellcell adhesion sits.

Integrins are the transmembrane protein at the focal adhesion that is involved in the assembly of the adhesion structure to which signaling molecules are incorporated. There are several cell adhesion molecules at the lateral cell adhesion site, but none was implicated to play a role in mechanosignal transduction. Our recent studies indicate, however, that PECAM-1 (platelet endothelial cell adhesion molecule1) appears to be a mechanoresponsive molecule.

\section{PECAM-1}

PECAM-1, also called CD31 and endoCAM, is a cellcell adhesion molecule most abundantly expressed in endothelial cells. It is also expressed by platelets, monocytes, neutrophils, and a certain subset of $\mathrm{T}$ lymphocytes (Newman et al., 1990). In cultured endothelial cells, PECAM-1 is diffusely distributed in the plasma membrane of solitary cells, but once a cell-cell contact is made, it accumulates at the contact site. In endothelial cells forming a confluent monolayer, it is exclusively localized to the cellcell border. PECAM-1 is thought to play an important role in forming and maintaining the contact inhibited state of endothelial cells in culture as monolayer formation is inhibited by antibodies against the external portion of PECAM-1 (Albelda et al., 1990). In vivo, it is believed to mediate transmigration of white blood cells through the endothelium (Vaporciyan et al., 1993; Nakada et al., 2000).

PECAM-1 was first cloned from human umbilical vein endothelial cells (Newman et al., 1990), and subsequently from mouse (Xie and Muller, 1993) and swine (Newman et al., 1992). Bovine PECAM-1 was cloned in our laboratory (Osawa et al., 1997). At the amino acid level, bovine PECAM-1 has $71 \%$ and $63 \%$ identity, and $80 \%$ and $77 \%$ similarity with human and mouse PECAM-1, respectively. It belongs to the immunoglobulin (Ig) superfamily and has 6 loops of the $\mathrm{C} 2$ type Ig-like domains, constituting a large extracellular portion of the molecule. It has a single transmembrane domain and a short cytoplasmic tail. The cytoplasmic domain of bovine PECAM-1 consists of 118 amino acids (Osawa et al., 1997). The primary structure of this domain is better preserved than that of the rest of the molecule among PECAM-1s from different species. The sequence homology is particularly high in the 40 or so of amino acids following the transmembrane domain (Osawa et al., 1997), suggesting the functional importance of this part of the molecule. 


\section{Tyrosine phosphorylation of PECAM-1}

The cytoplasmic domain of PECAM-1 contains 12 serine, 4 threonine, and 5 ( 6 in the case of bovine) tyrosine residues. Of these phosphorylatable amino acids, only serine phosphorylation was reported earlier in thrombin stimulated platelets (Newman et al., 1992). Phosphorylation of other residues was not reported, although the sequence data suggested that two tyrosines (tyr663 and tyr686) might be phosphorylated. We have shown that tyr686 is phosphorylated in endothelial cells that are stimulated by fluid shear stress of more than $5 \mathrm{dyn} / \mathrm{cm}^{2}$, or hyper- and hypo-osmotic shocks (Osawa et al., 1997). Recently, PECAM-1 tyrosine phosphorylation was detected in aggregating platelets (Jackson et al., 1997) and in mast cells (Sagawa et al., 1997).

Such PECAM-1 tyrosine phosphorylation in mechanically stimulated endothelial cells is a rapid event, detectable within 30 seconds of stimulation (Harada et al., 1995; Osawa et al., 1997). Although this is a rapid response, there are other flow-induced responses, such as a transient cytoplasmic $\mathrm{Ca}^{2+}$ increase (Ando et al., 1988) and $\mathrm{K}^{+}$channel activation (Olsen et al., 1988), that occur much faster than the PECAM-1 response. Thus, it is possible that the PECAM-1 phosphorylation is a downstream event of these faster responses. To investigate whether or not PECAM-1 tyrosine phosphorylation could be induced by simply increasing cytoplasmic $\mathrm{Ca}^{2+}$ concentrations, we treated endothelial cells with thrombin, ATP, histamine, bradykinin, or acetylcholin, all of which are known to increase the cytoplasmic $\mathrm{Ca}^{2+}$ concentration, or with calcium ionophores. None of these treatments was able to induce PECAM-1 tyrosine phosphorylation. $\mathrm{Ba}^{2+}$ and TEA (tetraethyl ammonium ion) have been shown to inhibit flow-elicited $\mathrm{K}^{+}$channel activities (Olsen et al., 1988), but they failed to block PECAM-1 tyrosine phosphorylation in osmotically stimulated endothelial cells. $\mathrm{Gd}^{3+}$, a stretch activated cation channel blocker, also failed to inhibit this PECAM-1 tyrosine phosphorylation. Activating protein kinase $\mathrm{C}$ (PKC) by PMA (phorbol myristate acetate) did not cause the PECAM-1 phosphorylation. These results strongly suggest that mechanically induced tyrosine phosphorylation of PECAM-1 is not a downstream event of $\mathrm{Ca}^{2+}$ mobilization, $\mathrm{K}^{+}$channel activation, stretch-activated cation channel activity, and PKC activation. We propose that PECAM-1 tyrosine phosphorylation is a unique response elicited in endothelial cells by mechanical forces and that it is a step involved in a novel mechanosignaling pathway in these cells.

\section{PECAM-1 and signal transduction}

SHP-2 (also known as Syp, SH-PTP2, PTP2C and PTP1D) is a homologue of Drosophila tyrosine phosphatase corkscrew (Freeman et al., 1992) which, when activated, transduces a positive signal to the MAP kinase pathway (Perkins et al., 1992). In mammalian cells, SHP-2 activates the ERK1/2 cascade (Noguchi et al., 1994). It binds to PECAM-1 only when the latter is tyrosine-phosphorylated (Masuda et al., 1997), and the similar binding activity was reported in platelets (Jackson et al., 1997). Based on these and other data (Tang et al., 1995), we have hypothesized that ERK activation, which is one of the well-known responses of endothelial cells to fluid shear stress (Takahashi and Berk, 1995), is an event downstream of PECAM-1 tyrosine phosphorylation and SHP-2 activation.

In confluent endothelial cells, PECAM-1 is localized to the cell-cell border and its localization does not change before and after tyrosine phosphorylation by mechanical stresses. SHP-2 is a cytoplasmic protein, distributed throughout the cytoplasm of unstimulated endothelial cells. However, shortly after cells are challenged with mechanical stresses (i.e. PECAM-1 is tyrosine-phosphorylated), antiSHP-2 immunofluorescence staining appears at the cell-cell adhesion site where PECAM-1 is localized (Masuda et al., 1997). This SHP-2 translocation represents SHP-2 binding to tyrosine-phosphorylated PECAM-1 in vivo. It is presumed that this association activates the phosphatase activity of SHP-2, which activates the Ras signaling pathway, leading to ERK activation.

Our results (Osawa et al., manuscript in preparation) indicate that when PECAM-1 expression in cultured endothelial cells was down-regulated by antisense S-oligonucleotides, the hyper-osmotically induced ERK activation was significantly reduced. In addition, this ERK activation was blocked by overexpressing phosphatase-nagative SHP2 in endothelial cells and mechanically stimulating them, even though PECAM-1 tyrosine phosphorylation occurred normally. PD 9809, a MEK inhibitor also blocked the ERK activation. These observations suggest that PECAM-1 tyrosine phosphorylation and SHP-2 activation are essential for activating the MEK-ERK pathway by mechanical stresses.

Although we have not investigated it in detail, Src family kinases may play important roles in the PECAM-1 mediated mechanosignal transduction. c-Src is known to be activated in endothelial cells by mechanical stresses (for review, see Traub and Berk, 1997). We have found that it can phosphorylate tyr686 of PECAM-1 in vitro and also binds to phosphorylated tyr686 of PECAM-1 via its SH2 domain (Osawa et al., 1997; Masuda et al., 1997). The Src family kinase is inactive when its $\mathrm{N}$-terminal $\mathrm{SH} 2$ domain is bound to its own phosphotyrosine residue at the $\mathrm{C}$-terminal tail. It becomes active when this intramolecular $\mathrm{SH} 2 /$ phosphotyrosine association is broken. There are two ways to achieve this. One is to dephosphorylate the tyrosine residue, and this can be achieved by the action of SHP-2 (Peng and Cartwright, 1995). Another way is for the SH2 domain to bind to a higher affinity $\mathrm{SH} 2$-binding motif on another protein (Pawson, 1995), and the phosphorylated tyr686 of PECAM1 may serve as a specific competitive binding site for the $\mathrm{SH} 2$ domain of the Src family kinase. It is quite possible 
that once PECAM-1 is tyrosine-phosphorylated, it may recruit and activate Src family kinases.

\section{PECAM-1 engagement and ERK activation}

PECAM-1 is exclusively localized at the cell-cell apposition site in confluent endothelial cells, but in sparsely cultured cells, anti-PECAM-1 stains the plasma membrane uniformly. We investigated if the formation of cell-cell adhesion (i.e. PECAM-1 engagement) was necessary for PECAM-1 tyrosine phosphorylation and ERK activation by mechanical stress. While PECAM-1 was phosphorylated in confluent cultures, its phosphorylation level in sparse cultures was significantly low. ERK, but not P38, activation by mechanical stress was also inhibited in sparse cultures. Although these data are not conclusive, they are consistent with the idea that PECAM-1 engagement is necessary for its tyrosine phosphorylation and subsequent signaling.

\section{PECAM-1 as a mechanosensing molecule}

Engaged PECAM-1 molecules are trapped in place so that mechanical force may be able to act on them. Thus, we investigated if direct force application onto PECAM-1 can cause its tyrosine phosphorylation. Magnetic beads were coated with antibodies against the external domain of PECAM-1 and applied onto sparse cultures of endothelial cells. PECAM-1 accumulated under the beads in a time dependent manner. By placing a strong magnet over the culture dish, attractive force was exerted to the magnetic beads. After 10 minutes of steady application of pulling force, the beads were isolated and the level of tyrosine phosphorylation of PECAM-1 associated with the beads was analyzed. We found that increased levels were detected only when a magnetic field was applied. Binding alone did not elevate the phosphorylation level. The experiments indicate that the tugging force applied to PECAM-1 molecules is responsible for PECAM-1 tyrosine phosphorylation. In addition, we found that ERK was also activated by the same procedure. Neither PECAM-1 tyrosine phosphorylation nor ERK activation occurred with magnetic beads coated with other molecules (such as non-immune IgG, and poly-D-lysine). These results suggest that PECAM-1 is a mechanoresponsive molecule and that its tyrosine phosphorylation leads to ERK activation (Osawa et al., manuscript in preparation).

\section{Perspectives}

Studies on signal transduction elicited by chemical stimuli have made rapid progress in recent years, but little advance has been made in terms of understanding the mechanism of mechanosignal transduction. One major difference between these two cases is the presence or absence of specific ligands. There is a general strategy for studying signaling by chemical stimuli. Once a ligand is identified, based on its affinity, one can expect to find a receptor for it. With mechanical stimuli, there is no ligand, and this makes it difficult to identify the molecule(s) responsible for sensing mechanical force. Studies on endothelial cell responses to fluid shear stress will benefit greatly if sensor molecules can be identified. PECAM-1 exhibits various characteristics that one expects for a mechanoresponsive molecule. To our knowledge, it is the first molecule in mammalian cells that is chemically modified when direct mechanical force is applied to it. It is hoped that PECAM-1 serves as a model system for analyzing mechanosignal transduction by mammalian cells.

The study on signal transduction involving PECAM-1 is still at its infant stage. Many questions remain to be answered, but some questions are more critical than others. One important question is how PECAM-1 is tyrosine-phosphorylated. Analyses of the primary structure of the PECAM-1 cytoplasmic tail reveal that the molecule does not appear to be an autophosphorylating protein. Thus, there must exist kinase(s) for PECAM-1. Our experiments show that direct application of mechanical force to PECAM-1 elicits its tyrosine phosphorylation. How would the kinase(s) get involved in this reaction? One possible mechanism is that force applied on PECAM-1 causes conformational changes in the molecule so that the tyrosine residues become exposed such the kinase(s) can rapidly phosphorylate them. Another question relates to the molecular link between PECAM-1 and stress fibers. Our preliminary results suggest an affinity between PECAM-1 and $\beta$-catenin. A third question is what the phosphorylation state of PECAM-1 is in in situ endothelial cells. How does it relate to the physiology of the endothelium? Our study (Kano et al., 2000) indicates that the level of phospho-tyrosine increases in the area of increased fluid shear stress in the blood vessel. In the light of the atheroprotective nature of high shear stress, PECAM-1 phosphorylation at the cell-cell junction may play a role in maintaining the healthy state of the endothelium.

Acknowledgments. The studies from our laboratory summarized in this review were supported by grants-in-aid for Scientific Research from the Ministry of Education, Culture, Sports, Science, and Technology of Japan, the Ministry of Health and Welfare of Japan, the Organization for Pharmaceutical Safety and Research (OPSR), and Special Coordination Funds of the Ministry of Education, Culture, Sports, Science and Technology of Japan.

\section{References}

Albelda, S.M., Oliver, P.D., Romer, L.H., and Buck, C.A. 1990. EndoCAM: a novel endothelial cell-cell adhesion molecule. J. Cell Biol., 110: 1227-1237.

Ando, J., Komatsuda, T., and Kamiya, A. 1988. Cytoplasmic calcium response to fluid shear stress in cultured vascular endothelial cells. In Vitro Cell Develop. Biol., 24: 871-877.

Bao, X., Lu, C., and Frangos, A.J. 1999. Temporal gradient in shear but not steady shear stress induces PDGF-A and MCP-1 expression in 
endothelial cells: role of NO, NF kappa B, and egr-1. Arterioscler. Thromb. Vasc. Biol., 19: 996-1003.

Buckley, C.D., Rainger, G.E., Bradfield, P.F., Nash, G.B., and Simmons, D.L. 1998. Cell adhesion: More than just glue. Mol. Memb. Biol., 15: $167-176$

Byers, H.R., White, G.E., and Fujiwara, K. 1984. Organization and function of stress fibers in cells in vitro and in situ. In Cell and Muscle Motility. R.M. Dawben and J.W. Shay, eds., Plenum Publishing Co., New York, pp. 83-137.

Caro, C.G., Fitz-Gerald, J.M., and Schrode, R.C. 1971. Atheroma and arterial wall shear. Observations, correlation and proposal of a shear dependent mass transfer mechanism for atherogenesis. Proc. Roy. Soc. Lond. (Biol.), 117: 109-159.

Davies, P.F. 1995. Flow-mediated endothelial mechanotransduction. Physiol. Rev., 75: 519-560.

Dejana, E. 1997. Endothelial adherens junctions: Implications in the control of vascular permeability and angiogenesis. J. Clin. Invest., 100: S7S10.

Dejana, E., Bazzoni, G., and Lampugnani, M.G. 1999. The role of endothelial cell-to-cell junctions in vascular morphogenesis. Thrombosis Haemastasis, 82: 755-761.

Dimmeler, S., Fleming, I., Fisslthaler, B., Hermann, C., Busse, R., and Zeiher, A.M. 1999. Activation of nitric oxide synthase in endothelial cells by Akt-dependent phosphorylation. Nature, 399: 601-605.

Dull, R.O. and Davies, P.F. 1991. Flow modulation of agonist (ATP) response $\left(\mathrm{Ca}^{2+}\right)$ coupling in vascular endothelial cells. Am. J. Physiol., 261: H149-H154.

Flaherty, J.T., Pierce, J.E., Ferrans, V.J., Patel, D.J., Tucker, W.K., and Fry, D.L. 1972. Endothelial nuclear patterns in the canine arterial tree with particular reference to hemodynamic events. Circ. Res., 30: 23-33.

Freeman, R.M., Plutzky, J., and Neel, B.G. 1992. Identification of a human src homology 2-containing protein tyrosine phosphatase: a putative homologue of Drosophila corkscrew. Proc. Natl. Acad. Sci. USA, 89: 11239-11243.

Fry, D.L. 1968. Acute vascular endothelial changes associated with increased blood velocity gradients. Circ. Res., 22: 165-197.

Gimbrone, M.A., Jr. 1999. Endothelial dysfunction, hemodynamic forces and atherosclerosis. Thrombosis Haemos., 82: 722-726.

Harada, N., Masuda, M., and Fujiwara, K. 1995. Fluid flow and osmotic stress induce tyrosine phosphorylation of an endothelial cell $128 \mathrm{kDa}$ surface glycoprotein. Biochem. Biophys. Res. Comm., 214: 69-74.

Helmke, B.P., Goldman, R.D., and Davies, P.F. 2000. Rapid displacement of vimentin intermediate filaments in living endothelial cells exposed to flow. Circ. Res., 86: 745-752.

Ishida, M., Peterson, T.E., Kovach, N.L., and Berk, B.C. 1996. MAP kinase activation by flow in endothelial cells: Role of integrin and tyrosine kinase. Circ. Res., 79: 310-316.

Jackson, D.E., Ward, C.M., Wang, R., and Newman, P.J. 1997. The protein-tyrosine phosphatase, SHP-2 binds platelet endothelial cell adhesion molecule-1 (PECAM-1) and forms a distinct signaling complex during platelet aggregation - Evidence for a mechanistic link between PECAM1 and integrin mediated cellular signaling. J. Biol. Chem., 272: 69866993.

Kano, Y., Katoh, K., Masuda, M., and Fujiwara, K. 1996. Macromolecular composition of stress fiber-plasma membrane attachment sites in endothelial cells in situ. Circ. Res., 79: 1000-1006.

Kano, Y., Katoh, K., and Fujiwara, K. 2000. Lateral zone of cell-cell adhesion as the major fluid shear stress-related signal transduction site. Circ. Res., 86: 425-433.

Kanzaki, M., Nagasawa, M., Kojima, I., Sato, C., Naruse, K., Sokabe, M., and Iida, H. 1999. Molecular identification of a eukaryotic, stretch-activated nonselective cation channel. Science, 285: 882-886.

Katoh, K., Masuda, M., Kano, Y., Jinguji, Y., and Fujiwara. K. 1995.
Focal adhesion proteins associated with apical stress fibers of human fibroblasts. Cell Motil. Cytosk., 31: 177-195.

Katoh, K., Kano, Y., Masuda, M., Onishi,, H., and Fujiwara, K. 1998. Isolation and contraction of the stress fiber. Mol. Biol. Cell, 9: 19191938.

Katoh, K., Kano, Y., and Fujiwara, K. 2000. Isolation and in vitro contraction of stress fibers. In Regulation and Effectors of Small GTPases. W.E. Blach, E.J. Der and A. Hall, eds. Methods in Enzymology, 325: 369-380.

Masuda, M., Osawa, M., Shigematsu, H., Harada, N., and Fujiwara, K. 1997. Platelet endothelial cell adhesion molecule-1 is a major SH-PTP2 binding protein in vascular endothelial cells. FEBS Lett., 408: 331-336.

Mo, M., Eskin, S.G., and Schilling, W.P. 1991. Flow induced changes in $\mathrm{Ca}^{2+}$ signaling of vascular endothelial cells: Effect of shear stress and ATP. Am. J. Physiol., 260: H1698-H1707.

Muller, J.M., Chilian, W.M., and Davis, M.J. 1997. Integrin signaling transduces shear stress-dependent vasodilation of coronary arterioles. Circ. Res., 80: 320-326.

Nagel, T., Resnick, N., Dewey, C.F., Jr., and Gimbrone, M.A., Jr. 1999. Vascular endothelial cells respond to spatial gradients in fluid shear stress by enhanced activation of transcription factors. Arterioscler. Thromb. Vasc. Biol., 19: 1825-1834

Nakada, M.T., Amin, K., Christofidou-Solomidou, M., O’Brien, C.D., Sun, J., Gurubhagavatula, I., Heavner, G.A., Taylor, A.H., Paddock, C., Sun, Q.H., Zehnder, J.L., Newman, P.J., Albelda, S.M., and DeLisser, H.M. 2000. Antibodies against the first Ig-like domain of human platelet endothelial cell adhesion molecule-1 (PECAM-1) that inhibit PECAM1-dependent homophilic adhesion block in vivo neutrophil recruitment. J.Immunol., 164: 452-462.

Newman, P.J., Berndt, M.C., Gorski, J., White, G.Cd., Lyman, S., Paddock, C. and Muller, W.A. 1990. PECAM-1 (CD31) cloning and relation to adhesion molecules of the immunoglobulin gene superfamily. Science, 247: 1219-1222.

Newman, P.J., Hillery, C.A., Albrecht, R., Parise, L.V., Berndt, M.C., Mazurov, A.V., Dunlop, L.C., Zhang, J., and Rittenhouse, S.E. 1992. Activation dependent changes in human platelet PECAM-1: Phosphorylation, cytoskeletal association, and surface membrane redistribution. $J$. Cell Biol., 119: 239-246.

Nilius, B., Viana, F., and Droogmans, G. 1997. Ion channels in vascular endothelium. Ann. Rev. Physiol., 59: 145-170.

Noguchi, T., Matozaki, T., Horita, K., Fujioka, Y., and Kasuga, M. 1994. Role of SH-PTP2, a protein-tyrosine phosphatase with Src homology 2 domains, in insulin-stimulated Ras activation. Mol. Cell Biol., 14: 66746682.

Ohtsuka, A., Ando, J., Korenaga, R., Kamiya, A., Toyama-Sorimachi, N., and Miyasaka, M. 1993. The effect of flow on the expression of vascular adhesion molecule-1 by cultured mouse endothelial cells. Biochem. Biophys. Res. Comm., 193: 303-310.

Okahara, K., Sun, B., and Kambayashi, J. 1998. Upregulation of prostacyclin synthesis-related gene expression by shear stress in vascular endothelial cells. Arterioscler. Thromb. Vasc. Biol., 18: 1922-1926.

Olsen, S.P., Clapnam, D.E., and Davies, P.F. 1988. Haemodynamic shear stress activates a $\mathrm{K}^{+}$current in vascular endothelial cells. Nature, 331: 168-170.

Osawa, M., Masuda, M., Harada, N., Lopes, R.B., and Fujiwara, K. 1997. Tyrosine phosphorylation of platelet endothelial cell adhesion molecule1 (PECAM-1, CD31) in mechanically stimulated vascular endothelial cells. Eur. J Cell Biol., 72: 229-237.

Pawson, T. 1995. Protein modules and signalling networks. Nature, 373: $573-580$.

Peng, Z.Y. and Cartwright, C.A. 1995. Regulation of the Src tyrosine kinase and Syp tyrosine phosphatase by their cellular association. Oncogene, 11: 1955-1962. 
Perkins, L.A., Larsen, I., and Perrimon, N. 1992. Corkscrew encodes a putative protein tyrosine phosphatase that functions to transduce the terminal signal from the receptor tyrosine kinase torso. Cell, 70: 225-236.

Sagawa, K., Swaim, W., Zhang, J., Unsworth, E., and Siraganian, R.P. 1997. Aggregation of the high affinity $\operatorname{IgE}$ receptor results in the tyrosine phosphorylation of the surface adhesion protein PECAm-1 (CD31). J. Biol. Chem., 272: 13412-13418.

Schilling, W.P., Mo, M., and Eskin, S.G. 1992. Effects of shear stress on cytosolic $\mathrm{Ca}^{2+}$ of calf pulmonary artery endothelial cells. Exp. Cell Res., 198: $31-35$.

Schwartz, G., Droogmans, G., and Nilius, B. 1992. Shear stress induced membrane curents and calcium transients in human vascular endothelial cells. Pflugers Archiv Eur. J. Physiol., 421: 394-396.

Shattil, S., and Ginsberg, M.H. 1997. Integrin signaling in vascular biology. J. Clin. Invest., 100: S91-S95.

Shen, J., Luscinskas, F.W., Connolly, A., Dewey, C.F., Jr., and Gimbrone, M.A., Jr. 1992. Fluid shear stress modulates cytosolic free calcium in vascular endothelial cells. Am. J. Physiol., 262: C384-C390.

Silkworth, J.B., McLean, B., and Stehbens, W.E. 1975. The effect of hypercholesterolemia on aortic endothelium studied en face. Atherosclerosis, 22: 335-348.

Sukharev, S.I., Blount, P., Martinac, B., Blattner, F.R., and Kung, C. 1994. A large-conductance mechanosensitive channel in E. coli encoded by mscL alone. Nature, 368: 265-268.

Takahashi, M. and Berk, B.C. 1996. Mitogen activated protein kinase (ERK1/2) activation by shear stress and adhesion in endothelial cells. Essential role for a hervinycine-sensitive kinase. J. Clin. Inv., 98: 26232631.

Tang, T.L., Freeman, R.M., Jr., O'Reilly, A.M., Neel, B.G., and Sokol, S.Y. 1995. The SH2-containing protein-tyrosine phosphatase SH-PTP2 is required upstream of MAP kinase for early Xenopus development. Cell, 80: 473-483.
Topper, J.N., Cai, J., Falv, D., and Gimbrone, M.A., Jr. 1996. Identification of vascular endothelial genes differentially responsive to fluid mechanical stimuli: Cyclooxygenase-2, manganese superoxide dismutase, and endothelial cell nitric oxide synthase are selectively up-regulated by laminar shear stress. Proc. Natl. Acad. Sci. USA, 93: 10417-10422.

Traub, O. and Berk, B.C. 1998. Laminar shear stress. Mechanism by which endothelial cells transduce an atheroprotective force. Arterioscler. Thromb. Vasc. Biol., 18: 677-685.

Tseng, H., Peterson, T.E., and Berk, B.C. 1995. Fluid shear stress stimulates mitogen activated protein kinases in bovine aortic endothelial cells. Circ. Res., 77: 869-878.

Ueba, H., Kawakami, M., and Yaginuma, T. 1997. Shear stress as an inhibitor of vascular smooth muscle cell proliferation. Role of transforming growth factor-beta 1 and tissue-type plasminogen activator. Arterioscler. Thromb. Vasc. Biol., 17: 1512-1516.

Vaporciyan, A.A., DeLisser, H.M., Yan, H.C., Mendiguren, I.I., Thom, S.R., Jones, M.L., Ward, P.A., and Albelda, S.M. 1993. Involvement of platelet-endothelial cell adhesion molecule-1 in neutrophil recruitment in vivo. Science, 262: 1580-1582.

Wang, N., Butler, J.P., and Ingber, D.E. 1993. Mechanotransduction across the cell surface and through the cytoskeleton. Science, 260: 1124 1127.

Xie, Y. and Muller, W.A. 1993. Molecular cloning and adhesive properties of murine platelet endothelial cell adhesion molecule. Proc. Nat. Acad. Sci. USA, 90: 5569-5573.

Zarins, C.K., Giddens, D.P., Bharadvaj, B.K., Sottiural, V.S., Mabon, R.F., and Glagov, S. 1983. Carotid bifurcation atherosclerosis. Quantitative correlation of plaque localization with flow velocity profiles and wall shear stress. Circ. Res., 53: 502-514.

(Received for publication, March 1, 2001

and accepted, March 1, 2001) 